\title{
Characterization of the Electrode-Skin Impedance of Textile Electrodes
}

\author{
Cristina C. Oliveira, José Machado da Silva \\ INESC TEC \\ Faculdade de Engenharia da Universidade do Porto, Portugal \\ Email: cristina.oliveira@fe.up.pt, jms@fe.up.pt
}

\author{
Isabel G. Trindade, Frederico Martins \\ R\&D Unit Textile and Paper Materials \\ Universidade da Beira Interior, Portugal \\ Email: itrindade@ubi.pt, fredjmartins@hotmail.com
}

\begin{abstract}
Wearable systems are expected to contribute for improving traditional biopotential signals monitoring devices due to higher freedom and unobtrusiveness provided to the wearer. Textile electrodes present advantages compared with the conventional $\mathrm{Ag} / \mathrm{AgCl}$ electrodes for the capturing of biopotentials, namely in terms of skin irritation due to the hydrogel and the need of a technician to place the electrodes on the correct positions. Due to the lack of hydrogel, textile electrodes present different electrical contact characteristics. The skinelectrode impedance is an important feature since it affects the captured signal quality. Although a low impedance is desired, a comfortable wearable system should not require the electrodes to be covered by the hydrogel or be moistened. A forearm sleeve provided with textile electrodes was used to study the electrode-skin impedance and the signal-to-noise ratio (SNR) of surface electromyographic (EMG) signals on a long-term use basis. The sleeve can be adjusted for different levels of tightening to control the pressure applied on the electrodes. The obtained results provide valuable information on the pressure that the textile garments of a sleeve or vest should apply on the recording electrodes, in order to assure a good electrical and mechanical contact between the electrodes and the skin and decrease the noise due to motion. It was observed that the electrode-skin impedance measurement alone is not sufficient to establish a relation with the SNR. The extraction of parameters from an electrical equivalent model of the electrode-skin interface allows to determine a relation with the model parameters and the SNR. The evaluation of these parameters during long-term monitoring will allow assessing the quality of biopotential measurements in textile electrodes.
\end{abstract}

\section{INTRODUCTION}

Most electrode based medical equipment perform a verification of the contact impedance prior to a measurement. Portable verifiers are available which physicians use for that purpose. The fast pace evolution of personal monitoring technology is affecting the traditional approach towards electrode utilization, in particular when considering extended monitoring periods. Careful skin preparation, electrode positioning and continuous verification, which most healthcare personnel are accustomed to, are not readily applicable to certain subjects, such as elderly, allergenic and pediatric [1], and for the case of personal monitoring technology, might not even represent an alternative; moreover, variations of the electrode-skin interface impedance are to be expected. When considering athlete's performance, daily activities monitoring, and other scenarios where the individuals will have to position the electrodes themselves or the electrodes are integrated within a garment (as in the case of textile electrodes), careful positioning and skin preparation cannot be considered a part of the procedure.

Textile electrodes present several advantages compared with the conventional silver/silver chloride $(\mathrm{Ag} / \mathrm{AgCl})$ type electrodes. They are flexible, unobtrusive, do not cause skin irritation due to the gel, and are a good candidate for chronic applications. Moreover, unlike $\mathrm{Ag} / \mathrm{AgCl}$ electrodes, do not need to be changed in long-term applications. However, the textile electrodes come in different shapes, materials and formats. The biopotentials recorded with these new electrodes might not be comparable in terms of signal quality with the gold standard of gel electrodes, but it has been shown that still good results can be obtained. However, their behavior has not been fully studied to properly understand how they affect the captured signal quality.

For example, experiments performed on human skin are difficult to analyze since the conductivity properties of human skin differ for each person and can change within hours. Also, since no adhesive forcing the contact with the skin is used, textile electrodes are subject to higher levels of noise and artifacts caused by motion and loosening contact.

Beckmann et al. [2] developed setups to characterize textile electrodes focusing on the contact impedance between the electrode and skin, and to characterize the textile conductors. The objective of their work was to measure the complex impedance of textile fabrics and to explore the influence of pressure, humidity, anatomical shapes and stretch.

The electrode-skin impedance is often modeled with the equivalent circuit shown in Fig. 1 [3]. The voltage source $E_{h c}$ models the half cell potential, capacitance $C_{d}$ is related with the electrical charge accumulated between the electrode and the skin, $R_{d}$ is the resistance between the electrode and the skin during the charge transfer, and $R_{s}$ is related with the electrolyte gel, sweat and the underlying skin tissue.

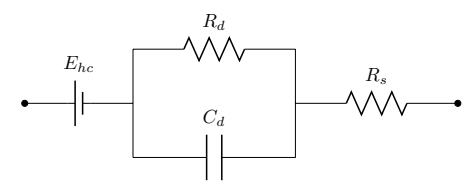

Fig. 1: Electrical model of the electrode-skin impedance. 


$$
Z(j \omega)=R_{s}+\frac{R_{d}}{1+j \omega R_{d} C_{d}}
$$

Besides the electrode-skin contact impedance dependency with the signal frequency, it also depends on the exerted pressure which can be considered in the impedance equation 1 after the use of pressure dependent $R_{d}$ and $C_{d}$ elements. Albulbul et al. [4] studied the effect of an externally applied pressure on $\mathrm{Ag} / \mathrm{AgCl}$ electrodes. It was concluded that a light to moderate applied pressure can decrease the electrodeskin impedance, but a large one may result in an impedance increase. According to the authors, this increase may be caused by a redistribution of the electrolyte used with nonpolarizable electrodes. Mihajlovic et al. [5] also studied the pressure effect in the electrode-skin interface of $\mathrm{Ag} / \mathrm{AgCl}$ and gold plated flat dry electrodes for electroencephalographic (EEG) monitoring. It was concluded that after applying a force, both the $\mathrm{Ag} / \mathrm{AgCl}$ and gold-plated electrodes present lower resistances and higher capacitances. Taji et al. [6] studied the effect of applied pressure in electrode-ski impedance for conductive textile electrodes. By increasing the pressure, the impedance magnitude decreased, as well as modeled resistors $R_{d}$ and $R_{s}$, and the capacitance $C_{d}$ increased. These studies obtained different impedance values for different electrodes, and even observed distinct behaviour for the same electrodes.

The electrode-skin impedance is known to affect the measured biopotentials, namely the noise level. Puurtinen et al. [7] measured the impedance and noise of surface electromyographic (EMG) signals captured with dry textile electrodes, textile electrodes moistened with water, and textile electrodes covered with hydrogel, for five different electrode sizes. The authors noted that the noise level increases as the electrode size decreases. The noise level obtained with dry textile electrodes was high, but that obtained with wet textile electrodes was low and similar to the noise level obtained with textile electrodes covered with hydrogel. The authors also found that hydrogel did not seem to improve noise properties, however it may have effects on movement artifacts. In this study they concluded that it is feasible to use textile embedded sensors in physiological monitoring applications when moistening or hydrogel is applied.

Taji et al. [6] developed a hardware-based configuration for acquiring electrocardiographic (ECG) signals with conductive textile, using various types of electrodes from different locations on the body. This was done to evaluate the overall quality of the acquired ECG based on the electrode-skin impedance measurements. The authors showed that the electrode-skin interface influences the quality and shape of the ECG signal registered at the electrode, and used the measured impedance to reconstruct the ECG signal.

The objective of the work reported in this paper is to study the influence of the pressure applied to the electrode-skin interface of embroidered textile electrodes with no hydrogel or moistened water on a long-term basis, i. e., after observing the variation of the electrode-skin impedance components over time. Measurements of EMG were also performed si- multaneously with the purpose of studying the influence of the impedance in the recorded biopotentials. The electrodeskin interface impedance is known to be related with the quality of the biopotential recordings. A mismatch between the electrode-skin impedances causes a degradation in the common mode rejection ratio (CMRR) and an increase in the power line frequency interference [8]. Some commercial ECG monitors include a test named lead-off to check the connectivity of an electrode to a patient. This test allows to verify if electrode is completely disconnected or when the connection is weak. However it is limited to the measurement of the impedance magnitude in just one frequency, and the lead-off threshold as to be defined by the system developer, and might differ for different electrodes [9]. There is a need to perform a test, based on the electrode-skin impedance, that is able to check the biosignal quality, and if possible correct the distorted signal. The relation between the impedance and the biosignal is not clear and needs to be further studied for textile electrodes.

Section II describes the procedures and methods adopted to measure the EMG signal with the textile electrodes, as well as the impedance. The acquired EMG data was processed to compute the SNR, and the impedance data was analyzed and the parameters from the equivalent electrical model of electrode-skin impedance were extracted using a data fitting algorithm. These operations are described in section III. The results obtained are discussed in section IV and conclusions are presented in section $\mathrm{V}$.

\section{Methods}

An elastic and adjustable forearm sleeve made of neoprene was realized at UBI for measurements of bio impedance and electromyography under controlled applied pressure on the region of the electrodes (see Fig. 2). The sleeve integrated two snap fasteners spaced apart by $4 \mathrm{~cm}$, for attachment of gel or dry electrodes and a textile pressure sensor. Textile electrodes having a circular a shape and $16 \mathrm{~mm}$ of diameter and integrating snap fasteners for attachment were realized using a 6-needle digital embroidery machine and electrically conductive threads Silverpam 250 from Tibtech having a resistivity of $200 \Omega \mathrm{m}^{-1}$ [10]. The textile pressure sensor was realized with embroidery processes and a plain weave fabric of bamboo with $30 \%$ silver from Less EMF Inc., Velostat and polyester foam. The sleeve allows the adjustment with Velcro of four tightening positions being the corresponding applied pressure on the electrodes region monitored with the pressure sensor.

The skin impedance is affected by temperature and total body water content [6]. In order to avoid detecting changes in the measured impedance that were not related to the electrodeskin impedance variations over time, all the measurements were performed in a room kept at a constant temperature. During the impedance measurements the test subject did not ingest any food or beverage. Prior to the measurements, the volunteer also went through skin preparation (skin was cleaned with alcohol). 


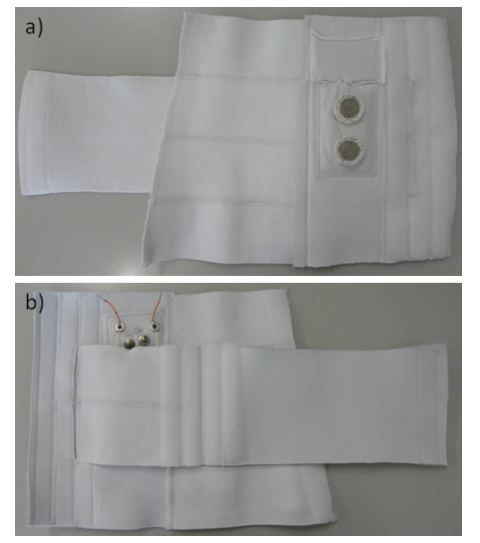

Fig. 2: Partial views of the forearm sleeve: a) inner part with textile electrodes b) outer part with a pair of snap fasteners to connected the data acquisition terminals and a pair of wires connected to the pressure sensor.

The textile electrodes pair was placed on the surface of the flexor carpi radialis muscle to acquire the EMG signal, and a standard $\mathrm{Ag} / \mathrm{AgCl}$ electrode with $1 \mathrm{~cm}$ diameter (Dormo, Telic S.A., Spain) was placed on the elbow to establish the reference.

\section{A. Electrode-Skin Impedance Measurements}

The impedance was measured for all the levels of tightening of the sleeve, in order to know which value of pressure ensures the best results, i. e., the lower levels of contact impedance.

The electrode-skin impedance was measured right after placing the sleeve, followed by the measurement of the EMG. This protocol was repeated after 15 minutes, after 30 minutes, and then every half an hour for two hours.

The impedance measurement was performed with the circuit described in [11]. A two-electrode configuration was used, with which a $10 \mathrm{~Hz}$ to $100 \mathrm{kHz}$ current sinewave (100 frequency steps in a logarithmic scale) signal was injected into one electrode and the voltage drop between the two electrodes was measured. This method allows the measurement of the impedance of the electrode pair plus the skin and body impedance. Since we want to study the electrode-skin interface of a single electrode, the measured impedance was divided by two, considering the two electrodes are identical. The measured impedance curves (magnitude and phase) were fitted to equation 1 using the least squares method.

A PCB (printed circuit board) with discrete components, creating the circuit in Fig. 1 with values in the range expected for the electrode-skin impedance, was utilized to perform the initial impedance measurements in order to test the accuracy of the impedance acquisition circuit and the fitting algorithm for the extraction of the circuit model parameters for the electrodeskin interface.

Table I presents the values of the PCB components, the extracted values from the fitting of the impedance measurements, and the relative error. The error is within the components tolerance. This measurement allowed to validate the impedance measurement circuit and the fitting algorithm.
TABLE I: Circuit parameters extraction using the impedance measurement.

\begin{tabular}{|c|c|c|c|}
\hline Parameters & $R_{s}(\Omega)$ & $R_{d}(\mathrm{k} \Omega)$ & $C_{d}(\mathrm{nF})$ \\
\hline Nominal & 1000 & 100.0 & 47 \\
\hline Fitted & 1049 & 114.8 & 41.07 \\
\hline Error (\%) & 4.88 & 14.78 & 12.61 \\
\hline
\end{tabular}

\section{B. Electromyography Measurements}

The EMG signal was acquired with a Biopac MP35 (Biopac Systems Inc, USA) with a sampling frequency of $2 \mathrm{kHz}$, and a bandpass filter between $5 \mathrm{~Hz}$ to $200 \mathrm{~Hz}$. During the first measurement the volunteer was asked to hold a hand dynamometer connected to the Biopac (SS25LA, BIOPAC Systems Inc., CA, USA) and clench it with the maximum force during maximum of 5 seconds. This was done three times, with 1 minute rest interval. The maximum value of force recorded was registered as the maximum voluntary contraction (MVC). During the next measurements the volunteer was asked to hold the hand dynamometer until $25 \%$ of the MVC for 5 seconds. This ensured that the EMG signal was acquired in the same circumstances and the SNR calculations are valid.

After the measurements of the EMG and clench force the SNR was estimated. For that purpose the recorded EMG signal was processed using Matlab R2013b (MathWorks Inc, MA, USA). First the DC component of the EMG signal was removed by subtracting its mean value. The signal was then rectified and smoothed with an envelope filter (cut-off frequency of $6 \mathrm{~Hz}$ ). The measured force was normalized by the MVC. The processed EMG and force signals are displayed in Fig. 3
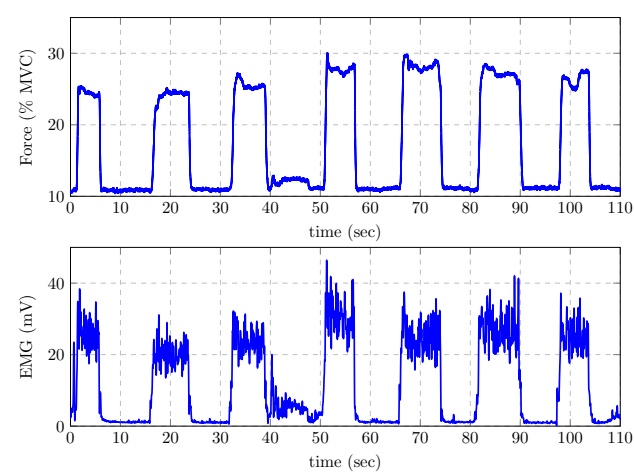

Fig. 3: Top: Force (normalized to the MVC) made by the volunteer when holding the hand dynamometer; Bottom: amplitude of the acquired EMG signal.

$$
S N R=20 \log _{10}\left(\frac{A_{\text {signal }}}{A_{\text {noise }}}\right)
$$

The EMG segments with activity that corresponded to a force of $25 \%$ of the MVC were chosen as the signal component, picking a length of 3 seconds.

The rms value of that signal was registered and the same procedure was used for the noise during a period of no activation of the muscles. The SNR is a ratio between the 
signal $\left(A_{\text {signal }}\right)$ and noise $\left(A_{\text {noise }}\right)$ amplitudes (see equation 2).

\section{RESULTS}

\section{A. Effect of Pressure on Electrode-Skin Impedance}

The electrode-skin impedance was measured with four different values of pressure applied to the electrodes. The highest value of pressure produced the lowest interface impedance. Fig. 4 displays the variation of the impedance magnitude with the frequency.

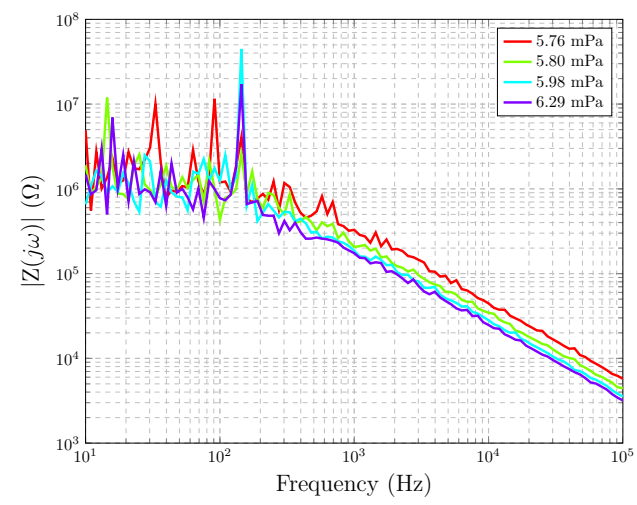

Fig. 4: Variation of the measured impedance magnitude with pressure and frequency.

The measured impedance curves (magnitude and phase) were fitted to equation 1 using the least squares method for each applied pressure. The values obtained for the model components are presented in table II. The $C_{d}$ capacitance is given by $C_{d}=\varepsilon \frac{A}{d}$, with $\varepsilon$ being the dielectric constant of the material between the electrode and the skin, $A$ is the area of the parallel plates that comprise the electrode, and $d$ is the distance between the plates, in this case the electrode and the skin. As the applied pressure increases, the effective contact area of the electrode increases (notice that the surface of the textile electrode is irregular) and the distance between the electrode and the skin decreases, which leads to a higher capacitance. The resistances $R_{d}$ and $R_{s}$ are given by $R=\rho \frac{L}{A}$, where $\rho$ is the resistivity of the material and $L$ the length. If were consider that the main varying factor is the contact area, a decrease in the resistances values is expected with an increase of the applied pressure. However, our observations show that this behavior is not linear, most likely because the sweat that is naturally produced and the humidity formed in the arm sleeve change the resistivity in the media between the electrode and the skin.

TABLE II: Equivalent circuit model parameters for the electrode-skin impedance for different applied pressures.

\begin{tabular}{|c|c|c|c|}
\hline Pressure $(\mathrm{mPa})$ & $R_{s}(\mathrm{k} \Omega)$ & $R_{d}(\mathrm{M} \Omega)$ & $C_{d}(\mathrm{nF})$ \\
\hline 5.76 & 6.75 & 2.11 & 0.30 \\
\hline 5.80 & 5.22 & 3.41 & 1.99 \\
\hline 5.98 & 3.94 & 1.12 & 2.96 \\
\hline 6.29 & 3.89 & 1.37 & 17.19 \\
\hline
\end{tabular}

The following measurements were performed with the sleeve tightened with the highest level, in order to accomplish a lower electrode-skin impedance, and therefore a better signal quality.

\section{B. Variation of Electrode-skin Impedance and SNR During Log-term Monitoring}

To evaluate long-term effects, impedance as well as EMG measurements were performed over time in four volunteers. Due to hardware restrictions the impedance and the EMG were not measured simultaneously, but both measurements were taken within less than 1 minute interval. All the volunteers performed their daily activities in the along the measurement period. The measurements for volunteer\#1 were taken 3 month before the measurements performed with the remaining volunteers. The impedances (magnitude and phase) obtained over time for all the volunteers are illustrated in Fig. 5- 8.
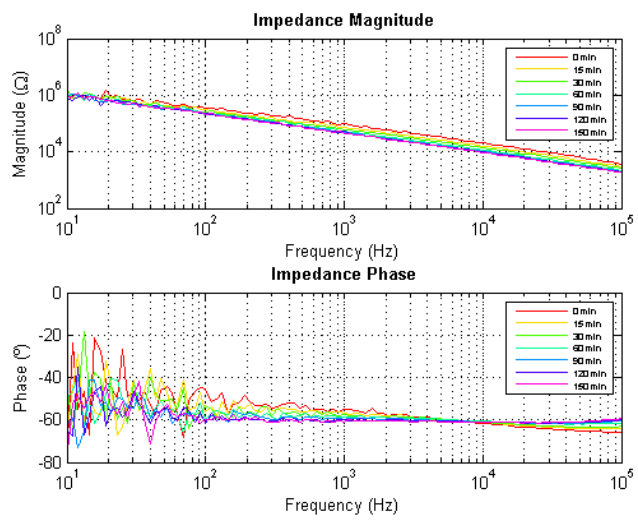

Fig. 5: Measured impedance for volunteer\#1.
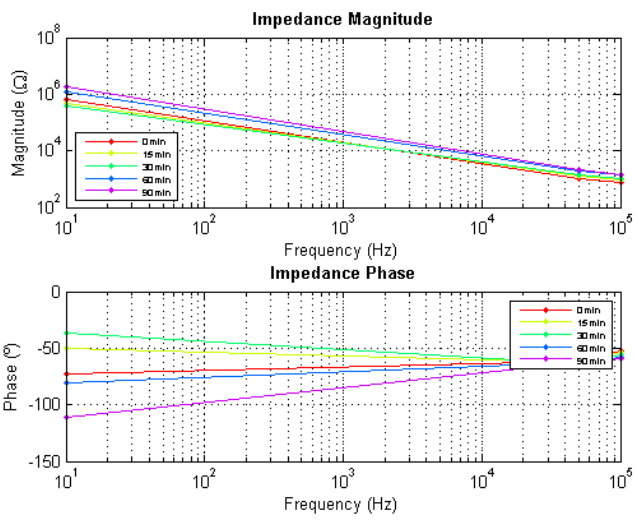

Fig. 6: Measured impedance for volunteer\#2.

The parameters for the electrode-skin impedance equivalent circuit (see Fig. 1) were extracted, and the SNR was calculated for each measurement. The variation of the impedance parameters $R_{s}, R_{d}$ and $C_{d}$ are plotted for all the measurement sessions with the volunteers (Fig.9 to 12). The evolution of the SNR is also included in the plots to provide a better insight 

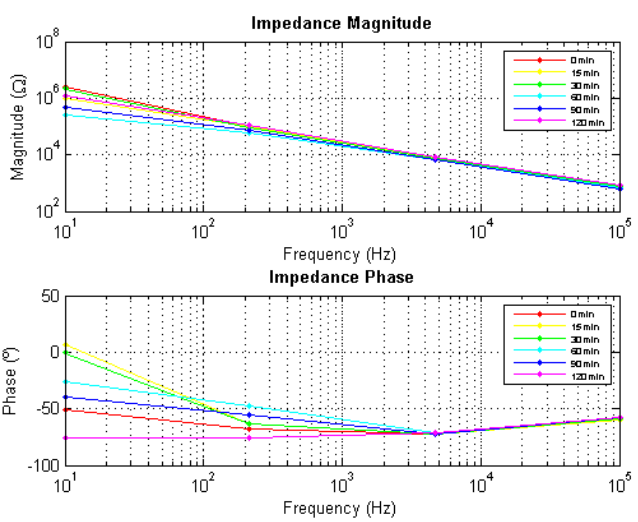

Fig. 7: Measured impedance for volunteer\#3.
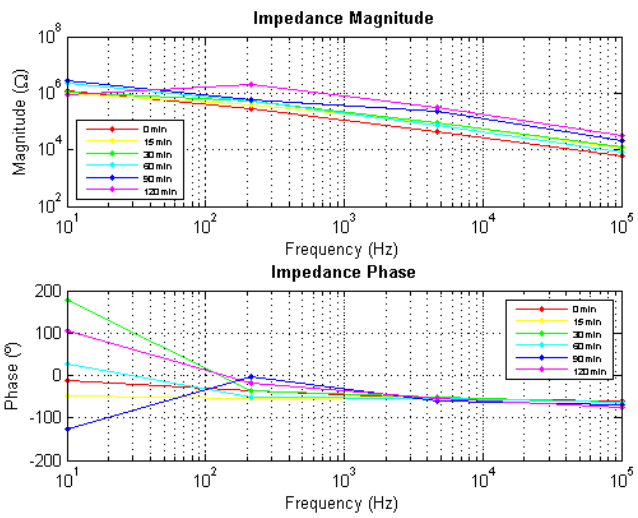

Fig. 8: Measured impedance for volunteer\#4.

of the parameters evolution and their influence on the signal quality.

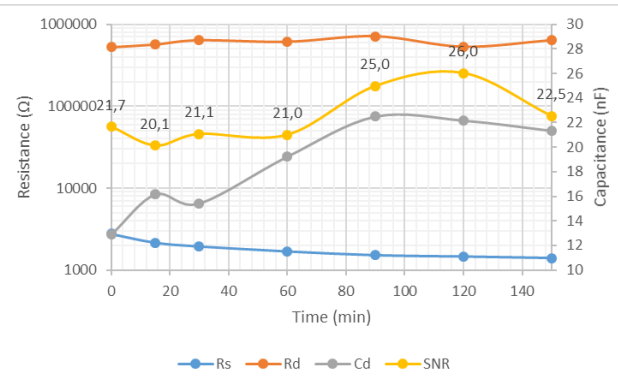

Fig. 9: Variation of $R_{s}, R_{d}, C_{d}$ and SNR for volunteer\#1.

\section{DISCUSSION}

The electrode-skin impedance measurements were carried out during long-term monitoring and evaluated considering EMG measurements performed at the same time, with the same electrodes. These measurements allow to study the variations of the impedance of the electrode-skin interface and its influence on the acquired signal, namely the SNR.

After observing the values of the electrode-skin impedance over time one can notice that the impedance variation is

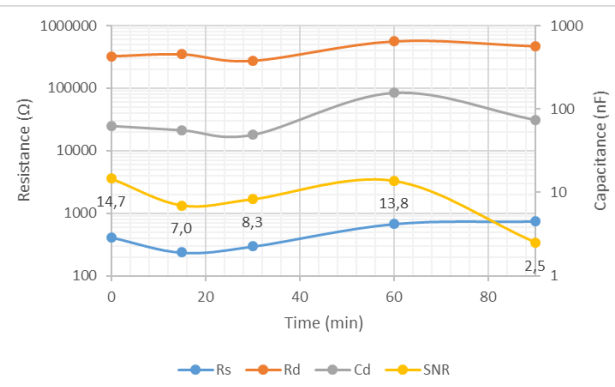

Fig. 10: Variation of $R_{s}, R_{d}, C_{d}$ and SNR for volunteer\#2.

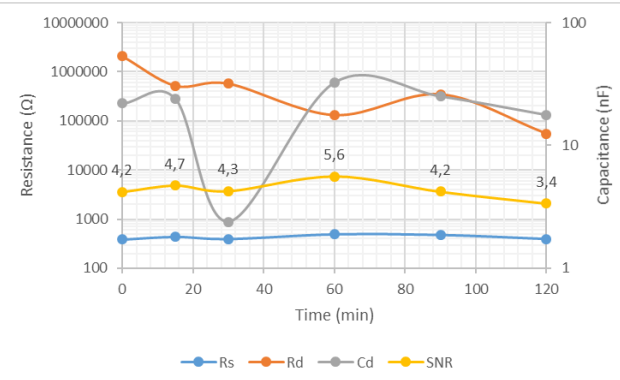

Fig. 11: Variation of $R_{s}, R_{d}, C_{d}$ and SNR for volunteer\#3.

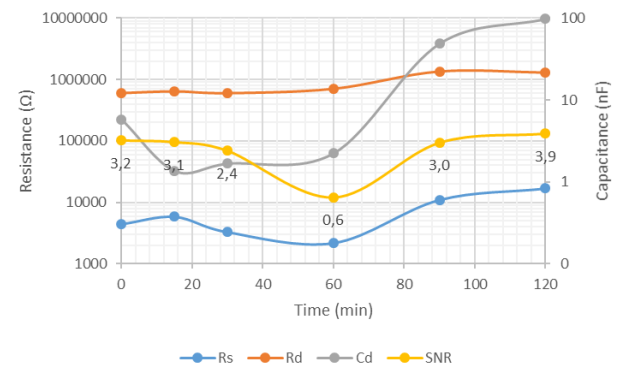

Fig. 12: Variation of $R_{s}, R_{d}, C_{d}$ and SNR for volunteer\#4.

very different among the volunteers. Although all of them were asked not to drink or eat anything during the measurements, they were free to perform their daily activities in the measurement period. During that time the hand sleeve and the electrodes might suffer small displacements, that could cause a shift in the impedance of the electrode-skin interface. From the observation of the impedance plots we could not see a relation with the SNR of the EMG signal. As the impedance measurements are influenced by the properties of the electrode/electrolyte and skin, we extracted the values of $R_{s}, R_{d}$ and $C_{d}$ to study their relation with the SNR individually.

If we hypothesize that the main factor for the SNR variation is the contact between the electrodes and the skin, the contact area would define how the impedance parameters would relate with the SNR. When the contact is better (higher effective area) the SNR and the $C_{d}$ should increase and the resistances $R_{d}$ and $R_{s}$ should decrease. Conversely, if the contact between the electrodes and the skin is loose the SNR and the $C_{d}$ 
decreases, and the $R_{d}$ and $R_{s}$ values increase. From the measurements of the impedance as a function of applied pressure (see Table II) we observed that the impedance parameters are indeed related with the electrode-skin contact and their values changed as expected, i.e. as the pressure increased the $C_{d}$ decreased, and both $R_{d}$ and $R_{s}$ decreased.

From Fig. 9 to Fig. 12 we notice the SNR is related with $C_{d}$ and $R_{s}$. When the SNR increases both $C_{d}$ and $R_{s}$ also increase, likewise for the reverse behavior. From our observations the $R_{d}$ has no relation with the SNR. Since these measurements were performed during an average of $2 \mathrm{~h}$, the electrode-skin interface changes (e .g., due to humidity in the sleeve), skin might produce sweet, and the electrode surface adapts to the skin. Also, the human body impedance of each person varies differently during the day. The $R_{d}$ could be more influenced by these parameters than the contact area itself. This study shows that an impedance magnitude measurement is not enough to monitor the quality of biosignals, since the resistance if affected by the person's own impedance variation. The extraction of the parameters of the electrodeskin impedance electrical model, after performing phase and magnitude measurements, is more reliable for the purpose of the signal quality monitoring.

A significant reduction of the SNR is observed comparing the measurements with the volunteer $\# 1$ and the remaining measurements. As mentioned before these measurements were performed 3 months apart. During this period the electrodes suffered aging, which is a known characteristic of textile electrodes.

In wearable devices the comfort is an important factor. In future measurements the volunteers should be given a questionnaire to assess the comfort of the sleeve and textile electrodes. Also, the optimum pressure applied to the garment should not be chosen solemnly based on the impedance values, but also on the feedback from the volunteers. If possible the values of applied pressure on the skin should be checked in the literature and/or with a physician to make sure the applied force does no affect the circulatory system.

To the best of our knowledge, this is the first study that relates the electrode-skin impedance and a biopotential (EMG) using textile electrodes without any hydrogel or water moistening. From our point of view the main advantages of textile electrodes are the no need for skin and electrode preparation, easy of use, and no need for using hydrogel. If the electrodeskin impedance is one of the factors that degrades the quality of the signal in textile electrodes, this parameter should be studied and compensated directly in the circuit and/or by means of post-processing.

\section{CONCLUSION}

The last decade has witnessed an explosion on the number of personal monitoring systems for different applications such as medical, rehabilitation, sports, and leisure. Electrical biosignals are commonly utilized within such systems and are traditionally captured with electrodes in their many forms. The electrode-skin interface is constantly changing, thus affecting the signal integrity and degrading at times the quality of the captured signal.

The work presented here relates the electrode-skin impedance and the EMG signal quality (SNR) obtained with textile electrodes with no hydrogel or water moistening. The authors observed that the analysis of either impedance magnitude or phase is not enough to monitor the biosignal quality. After extracting the parameters of electrode-skin impedance electrical model a relation between components values and the signal to noise ration could be achieved.

\section{ACKNOWLEDGMENT}

This work is being carried-out with the support of the ERDF - European Regional Development Fund through the COMPETE Programme (operational programme for competitiveness) and by National Funds through the FCT - Fundação para a Ciência e a Tecnologia (Portuguese Foundation for Science and Technology) within project SIVIC PTDC/EEI-ELC/1838/2012, and grant contract SFRH/BD/81476/2011 (first author).

The authors would like to thank Antonio J. Salazar (INESC TEC, FEUP) for his assistance with the impedance measurements, professor Miguel V. Correia from (INESC TEC, FEUP) for the support with the Biopac equipment, and Pedro Fonseca (LABIOMEP, Universidade do Porto) for the guidance in the acquisition and analysis of the EMG signal. The authors are also thankfull to the volunteers who participated in this study.

\section{REFERENCES}

[1] C. Assambo, A. Baba, R. Dozio, and M. J. Burke, "Determination of the parameters of the skin-electrode impedance model for ECG measurement," in Proceedings of the 6th WSEAS international conference on electronics, hardware, wireless and optical communications, Corfu Island, Greece, 2007, p. 9095.

[2] L. Beckmann, C. Neuhaus, G. Medrano, N. Jungbecker, M. Walter, T. Gries, and S. Leonhardt, "Characterization of textile electrodes and conductors using standardized measurement setups," Physiological Measurement, vol. 31, no. 2, p. 233, Feb. 2010.

[3] M. R. Neuman, "Biopotential electrodes," in The Biomedical Engineering Handbook, Second Edition. 2 Volume Set, J. Bronzino, Ed. CRC Press, Dec. 1999.

[4] A. Albulbul and A. Chan, "Electrode-skin impedance changes due to an externally applied force," in 2012 IEEE International Symposium on Medical Measurements and Applications Proceedings (MeMeA), May 2012, pp. 1-4.

[5] V. Mihajlovic and B. Grundlehner, "The effect of force and electrode material on electrode-to-skin impedance," in 2012 IEEE Biomedical Circuits and Systems Conference (BioCAS), 2012, pp. 57-60.

[6] B. Taji, S. Shirmohammadi, V. Groza, and I. Batkin, "Impact of skinelectrode interface on electrocardiogram measurements using conductive textile electrodes," IEEE Transactions on Instrumentation and Measurement, vol. 63, no. 6, pp. 1412-1422, Jun. 2014.

[7] M. Puurtinen, S. Komulainen, P. Kauppinen, J. Malmivuo, and J. A. K. Hyttinen, "Measurement of noise and impedance of dry and wet textile electrodes, and textile electrodes with hydrogel," in 28th Annual International Conference of the IEEE Engineering in Medicine and Biology Society, 2006. EMBS '06, 2006, pp. 6012-6015.

[8] T. Degen and H. Jckel, "Enhancing interference rejection of preamplified electrodes by automated gain adaption," IEEE transactions on biomedical engineering, vol. 51, no. 11, pp. 2031-2039, Nov. 2004.

[9] A. Calabria, "Understanding Lead-Off Detection in ECG," Texas Instruments, Tech. Rep., May 2012.

[10] I. G. Trindade, P. Spranger, F. Martins, R. Miguel, and M. S. Silva, "Fully integrated embroidery process for smart textiles," in Proc. of the Nanotech conference. Washington D.C., USA: Nanotech 2014, Jun. 2014, vol. 3, pp. 65-68.

[11] A. J. E. Salazar, J. Machado da Silva, M. Correia, and B. J. Mendes, "Built-in self-testing methodology and infrastructure for an emg monitoring sensor module," IARIA International Journal On Advances in Systems and Measurements, vol. 7, no. 1 \& 2, 2014. 